\section{DISCURSOS Y PRAXIS DE LA INNOVACIÓN EN ENTIDADES DE LA C.A. DEL PAÍS VASCO: UN ANÁLISIS APLICADO}

\author{
Auxkin Galarraga Ezponda \\ Álvaro Luna García \\ Sandra González Durán \\ Marcelino Massa Carrasquello \\ Departamento de Sociología II \\ Universidad del País Vasco
}

\section{DISCOURSES AND PRAXIS OF INNOVATION IN ENTITIES OF THE BASQUE COUNTRY: AN APPLIED ANALYSIS}

\begin{abstract}
This article is based on a project done by the research group "Change, Complexity and Innovation" that is part of the Association Society of Knowledge, Development and Innovation (ASCIDE), in an attempt to analyze the discourses and practices that surround the concept of innovation in different cultural, social, economic and institutional entities of the Basque Country. The main objective of this study is knowing how the concept of innovation is build and managed in the different entities. We work with the hypothesis that this concept is also used and understood outside its' entrepreneurial, scientific and technologic connotations, and therefore it is also important to know how the concept works in the cultural, institutional, social and educational spheres.
\end{abstract}

KEY WORDS: Innovation; discourse; practices; knowledge; research.

\section{INTRODUCCIÓN}

Frente a las múltiples voces que en la actualidad recurren al concepto de innovación en clave de respuesta a los dilemas de nuestra época, la investigación empírica que presentamos en las siguientes líneas cuestiona los recorridos que la innovación realiza en el momento de "tomar tierra" en los diferentes contextos en los que penetra. En la puesta en marcha de la investigación partimos desde la necesidad de extender la mirada científica más allá de los discursos sobre las bondades y posibilidades que brinda la innovación, para dirigirnos al análisis sobre las formas de comprensión en torno al concepto y los recorridos fluctuantes que emergen en las dinámicas innovadoras.

Para ello, el Grupo de Alto Rendimiento del Sistema Universitario Vasco "Cambio, Innovación y Complejidad So-
RESUMEN: El presente artículo está basado en una investigación realizada por el grupo de investigación "Cambio, complejidad e Innovación" perteneciente a ASCIDE (Asociación Sociedad del Conocimiento, Desarrollo e Innovación), en un intento por dilucidar los discursos y las prácticas que giran en torno al concepto de innovación dentro de las entidades de la Comunidad Autónoma del País Vasco. El objetivo principal de este estudio era conocer los entresijos que envuelven al concepto de innovación y a las formas en las que dicho concepto se emplea más allá de las connotaciones empresariales y científicas que dicho concepto ya posee, extendiendo, por tanto, los ámbitos de análisis del mismo a lugares vinculados con la cultura, lo social, lo institucional y lo educativo.

PALABRAS CLAVE: Innovación; discurso; prácticas; conocimiento; investigación.

cial" dirigido por Ander Gurrutxaga ha diseñado una investigación de dos fases ${ }^{1}$ con el análisis de los discursos y prácticas de los agentes concretos como hilo conductor. La primera fase se acerca, a través de entrevistas personales cualitativas, a las experiencias contadas por diversas entidades de la C.A. del País Vasco (empresas, asociaciones, fundaciones, colegios, colectivos sociales), que actúan en diversos ámbitos y sobre objetivos claramente diferentes, para así poder indagar tanto en los mecanismos que utilizan para intentar poner en práctica la innovación, como en las ambivalencias y paradojas que surgen en este proceso. De esta manera, de cara a la segunda fase se ha diseñado una encuesta para conocer la percepción social de la ciudadanía vasca en torno a las dinámicas con las que está funcionando la innovación en la actualidad, sobre qué ambitos consideran que se está aplicando y con qué agentes asocian la práctica innovadora. 
A través de estos dos análisis y conjugando técnicas cualitativas y cuantitativas, esperamos obtener un retrato ilustrativo de la interacción entre el ejercicio de la innovación y su percepción entre la ciudadanía. En esta ocasión, exponemos los resultados más destacados obtenidos en la primera fase de la investigación, en la que hemos examinado las formas en las que se intenta poner en práctica la innovación y las problemáticas que dicha experimentación genera en los ámbitos y agentes concretos que tratan de incorporarla a su vida cotidiana.

\section{LOS SENTIDOS DE LA INNOVACIÓN SOCIAL}

Somos conscientes de que nos encontramos ante un concepto -la innovación- que en fechas recientes ha adquirido una expansión y un protagonismo inusitado, convirtiéndose en un recurso para todo lo que tiene relación con el cambio, el aprendizaje, la experimentación, la transferencia de conocimiento y la acción de los grupos sociales, sobre todo en el ámbito empresarial y tecnocientífico. Estamos ante un concepto de moda, sobre el que lejos de avanzar una categorización operativa como parte de las herramientas analíticas que nos permitan comprender un mundo en modificación acelerada, caminamos hacia el incremento notable de la confusión e indeterminación en torno a los fenómenos que alberga. No obstante, no ha sido hasta hace bien poco cuando el concepto de innovación ha dejado de asociarse únicamente al ámbito científico, tecnológico y empresarial, ante la constatación de que su práctica también está presente en otros muchos ámbitos y contextos (Young Foundation, 2007; NESTA 2007), e incluso que la innovación tecnocientífica y empresarial es deudora de dinámicas socio-culturales, como el aprendizaje, la interacción, la confianza, el respeto, la creatividad, etc. (Von Hippel, 1998; Chesbrough, 2009, Lester y Piore, 2004).

Consideramos que la participación de las ciencias sociales en los estudios y análisis sobre la innovación está contribuyendo a una amplitud de miras sobre el proceso de innovación en muchos sentidos ${ }^{2}$. Los trabajos realizados desde la perspectiva de la innovación social y cultural han abierto importantes líneas de reflexión sobre los factores y dinámicas socio-culturales presentes en cualquier proceso de innovación, que van más allá del mero análisis de los efectos de la innovación tecnológica en la sociedad (Klein y Harrison, 2006). Dichos planteamientos nos recuerdan que la innovación se expresa en diferentes ámbitos (económico, empresarial, social, tecnológico, educativo, cultural), a través de componentes relacionales y sociales, donde dimensiones como el capital social, las redes, la interacción o la difusión adquieren un renovado protagonismo (Hämäläinen, 2007; Moulaert y Nussbaumer, 2005).

En nuestro caso, a la hora de realizar nuestra investigación, partimos de la perspectiva de la innovación social y cultural, entendida como óptica multidimensional y abierta desde la que aprehender las múltiples facetas y expresiones de la innovación que acontecen en los diversos ámbitos de la realidad social. Reseguimos la línea marcada por Ander Gurrutxaga (2010a: 133-134) cuando plantea que "la innovación es un proceso de cambio evolucionado en el conocimiento o en la acción en torno a cualquier dimensión social, política, cultural o económica de la vida", producido gracias a la "confluencia de múltiples actores sociales que participan los unos con los otros compartiendo información y conocimiento dentro de las redes sociales en las cuales el grado de participación o capital social es crucial para la mayor difusión de la red".

Desde este punto de partida, a la hora de llevar a cabo nuestra investigación sobre las dinámicas innovadoras en entidades de la C.A. del País Vasco hemos tenido en cuenta los siguientes aspectos y contextos que modelan los caracteres de la praxis de la innovación: a) ámbitos en los que la innovación se expresa, que pueden ser empresas y centros de investigación, pero también escuelas, organismos públicos, políticas sociales, espacios culturales o expresiones artísticas, solamente por citar algunos posibles; b) la dimensión, que en ocasiones llega a ser de carácter macro, pero viene precedida de innovaciones de carácter micro y meso; c) los procesos que están encadenados en la dinámica de la innovación y que suponen la presencia constante de la interdependencia; d) los mecanismos que posibilitan la difusión de las dinámicas innovadoras y los procesos de aprendizaje vinculados al mismo.

Desde este enfoque teórico consideramos que la praxis de la innovación social aparece ante nuestros ojos con cuatro características fundamentales. En primer lugar, consideramos que la innovación ha pasado a convertirse en un 
concepto polifónico y multidimensional, que retrata un fenómeno social que acontece en los diversos ámbitos de la realidad social. Así, debido a la elasticidad adquirida por el término, éste se asemeja, cada vez más, a un concepto contenedor (Gurrutxaga, 2010b: 52), que no necesita ser explicado y con la que todos los agentes y entidades quieren ser identificados, a pesar de la falta de reflexiones profundas sobre lo que verdaderamente significa ser innovador y las múltiples problemáticas que genera su puesta en práctica.

En segundo lugar, no podemos olvidar que la innovación, su práctica concreta, está inseparablemente unida a los contextos específicos y los rasgos socio-estructurales existentes (MacCallum, Moulert, Hillier y Vicari, 2009). Al contrario de muchos de los discursos existentes sobre la innovación que esconden las fricciones, problemas y dificultades que se generan a lo largo del proceso, nuestro planteamiento ha asumido de partida que las caracteristicas socio-estructurales de los espacios geográficos concretos plantean un cuadro de posibilidades y limitaciones que pueden ser modificados, gestionados y negociados, pero que no pueden ser evitados.

En esta misma línea, como tercera premisa consideramos que la innovación acontece en un entorno social articulado donde se generan múltiples y complejas experiencias de aprendizaje e imitación, así como procesos de transferencia de conocimientos, prácticas y habilidades que se dan tanto entre personas, como entre espacios geográficos y generaciones ${ }^{3}$. La creación y conexión a las redes sociales se hace posible gracias a la extensión de valores intangibles, tales como la confianza, la creatividad, el respeto, la colaboración, etc., que generan una cultura de la innovación cocinada a ritmos pausados que permea las diversas instituciones sociales y prácticas de los agentes (Saxenian, 1994).

Por último, la praxis de la innovación busca una orientación metodológica, esto es, la consolidación e institucionalización de buenas prácticas. Por ello, la práctica innovadora está siempre asociada al esfuerzo de implantación de metodologías, procedimientos, estrategias tanto formales como informales que deriven en la expansión de la innovación (Young Foundation, 2008). Sin embargo, la innovación no es un acontecimiento que se exprese de forma lineal y que genere resultados garantizados y siempre positivos. Muy al contrario, la innovación está atravesada por múltiples barreras, dificultades y paradojas que la convierten en un proceso complejo que, como toda praxis social, produce también consecuencias inesperadas e incalculables (Gurrutxaga, 2007).

Desde estos cuatro puntos fundamentales nos hemos acercado a las experiencias de praxis de la innovación en diversas entidades de la C.A. del País Vasco, con la intención de analizar: 1) el proceso de construcción de la conciencia innovadora; 2) los contextos socio-estructurales que delimitan las posibilidades y las limitaciones de la praxis innovadora de las diversas entidades; 3 ) los procesos de conexión que ejercen las entidades a las diversas redes en marcha y los recursos y aprendizajes que se derivan de la articulación; 4) los recorridos fluctuantes que realiza la innovación, desde los intentos de su institucionalización hasta las consecuencias inesperadas generadas en el camino.

\section{Planteamiento metodológico DE LA INVESTIGACIÓN}

Considerando, como venimos argumentando, que la innovación emerge en diferentes ámbitos y contextos, tratamos de recoger en nuestra investigación una variedad de experiencias que ofrecieran una primera imagen de las caracteristicas que adquiere la praxis de la innovación y cómo ésta es abordada en el quehacer cotidiano de las organizaciones.

La selección realizada de entre el amplio universo de las que practican la innovación o son identificadas como innovadoras en el País Vasco deriva de una exploración previa sobre qué actividades realizaban, cómo las realizaban y cómo ello era percibido por su entorno. De forma que lejos de analizar a las únicas entidades que están involucradas en dinámicas innovadoras y asumir entonces que la innovación se da y lo hace de cierta manera, desde nuestra perspectiva hemos optado por analizar a aquellas que de entre muchas otras son reconocidas o se apropian de forma expresa del concepto de innovación, tratando de recoger la variedad de experiencias que surgen cuando la innovación es abordada desde diversos ámbitos, agentes y dimensiones. 
De esta manera, completamos un total de 26 entrevistas en profundidad durante los meses de octubre y diciembre del año 2009 en entidades clasificadas en base a siete ámbitos diferenciados, desde la hipótesis de que la praxis de la innovación está condicionada y caracterizada tanto por el ámbito concreto en el que operan los agentes, como por la dimensión que las mismas adquieren, aunque también pueden distinguirse elementos transversales que permean sobre los diversos ámbitos y contextos, y que son el principal objeto de análisis en este texto.

Las entrevistas realizadas estaban clasificadas de la forma que sigue: dos entidades del ámbito industrial; tres entidades del ámbito tecnológico; cinco entidades del ámbito de los servicios; cinco entidades del ámbito cultural; tres entidades del sector institucional; cinco entidades del ámbito social; y por último, tres entidades del ámbito educativo. En cada uno de estos sectores hemos querido analizar la importancia que cada una de las entidades otorga a la innovación y al conocimiento, y a las formas de gestionar, organizar y desarrollar sus actividades y proyectos diarios.

Mediante el análisis de los diferentes relatos (análisis del discurso) aportados por las diversas entidades seleccionadas, hemos identificado una serie de pautas o patrones clave que posibilitan completar una reconstrucción de su propia comprensión de la innovación, así como las formas en las que han tratado de afianzar dinámicas innovadoras a lo largo de su trayectoria. Se utiliza el concepto de "reconstrucción" porque la entrevista en profundidad, técnica de investigación cualitativa utilizada en este estudio, fomenta entre las personas entrevistadas el propio hecho de mirar hacia atrás, buscando una coherencia o explicación lógica a decisiones que, posiblemente, no tuvieron ese mismo grado de conciencia en el momento de su puesta en práctica. Nos confrontamos, por lo tanto, a relatos reconstructivos sobre las trayectorias de entidades que tratan de integrar el discurso y la práctica de la innovación, en un proceso que genera nuevas posibilidades, pero donde también tienen cabida múltiples dificultades, ambivalencias y problemáticas.

\section{El ANÁlisIS DE LAS ENTREVISTAS EN PROFUNDIDAD}

El análisis en profundidad de los relatos que los informantes han realizado a lo largo del estudio sobre la forma en la que integran la praxis innovadora a la actividad cotidiana de sus organizaciones supone un notable contrapeso al discurso afirmativo y positivo con el que siempre es presentada la innovación. A la hora de pasar del discurso a la acción concreta, los informantes revelan las múltiples paradojas, dificultades, imprevistos y fluctuaciones que existen en el momento de institucionalización de la innovación. Ello nos conduce a considerar que la innovación es un proceso de largo recorrido que sufre diversos altibajos y provoca consecuencias no previstas. En esta situación, las diferentes organizaciones tratan de gestionar lo mejor que pueden la complejidad interna que genera la propia innovación, ya sea a través de planes estratégicos, evaluaciones, reflexiones más o menos formalizadas, etc., o incorporando criterios de adaptación permanente y de flexibilidad a sus propias organizaciones. Sin embargo, lejos de controlar el recorrido de la innovación y sus direcciones, la gestión de la innovación, acaba generando inevitablemente mayor complejidad.

De esta manera, difícilmente podremos afirmar que el recorrido de la innovación no tiene nunca un punto de llegada en el que toma una velocidad de crucero y genera resultados constantes. Muy al contrario, los relatos construidos por nuestros informantes muestran que la innovación es un recorrido cambiante en donde predomina la adaptación y la revisión constante de lo realizado hasta el momento. Las personas y las organizaciones no parecen tener dificultades para abrazar el discurso imperante que se ha extendido sobre la innovación, pero en el análisis realizado puede observarse que el conflicto surge a la hora de asumir con todas sus consecuencias lo que en la práctica significa ser innovador. Por ello, partimos de la necesidad de distinguir entre discurso (comprensión/percepción) y praxis de la innovación, de forma que podamos recomponer las piezas que integran el cuadro en torno a las paradojas y contingencias que genera la práctica de la innovación.

\subsection{La interiorización del discurso sobre la innovación y la construcción de la conciencia innovadora}

Uno de los grandes retos a los que la investigación que estamos exponiendo a través de este texto se ha enfrentado es la necesidad de superar la barrera creada por el "discurso dominante sobre la innovación" en las diversas entidades, para así poder indagar en los recorridos, posibilidades y limitaciones con los que se encuentra la práctica cotidiana de la innovación. A este respecto, a lo largo de 
las entrevistas hemos observado que las diversas entidades integran el discurso de la innovación a sus propios relatos y proclamas. Incluso, cómo varias de las entidades analizadas han asumido la etiqueta de entidades innovadoras otorgada desde el exterior, gracias a reconocimientos, premios o galardones recibidos por parte de agentes ajenos a la propia entidad. De esta manera, el conjunto de entidades analizadas asume el compromiso de avanzar en la implantación de dinámicas innovadoras, aunque sin poder explicar con claridad cómo ponen en práctica la innovación, cuáles son las medidas concretas que definen su desarrollo y, sobre todo, los beneficios y dificultades que ésta genera.

La innovación es identificada por las propias entidades entrevistadas con prácticas habituales en el desempeño cotidiano que mantienen una lógica continua desde hace mucho tiempo, antes incluso que el término innovación estuviese tan en boga de forma tan generalizada. En esta lógica, las personas informantes parecen querer indicar que el propio hecho de la supervivencia de la entidad es, en sí mismo, indicador suficiente para demostrar su capacidad de adaptación permanente y de incorporación de prácticas innovadoras. Con ello constatamos la existencia de una afinidad o asociación latente muy relevante entre innovación y supervivencia; y entre innovación y adaptación permanente, lo que implica que la innovación no sería más que el recurso necesario, aunque no suficiente, para el mantenimiento de una organización a lo largo del tiempo 4 .

En este sentido, el concepto de innovación es utilizado por las propias entidades de puertas para dentro para dar sentido a las actividades que se pretenden implantar, ofreciendo un soporte muy socorrido para la reestructuración y reorientación de diversas actividades y formas de organización $^{5}$. De puertas hacia fuera, en cambio, aquellas entidades que han conseguido ser etiquetadas como innovadoras abrazan el concepto, conscientes de que en la actualidad ello supone "un valor positivo", que otorga "una diferenciación", "un plus" dentro del conjunto de agentes, estructuras, procesos y redes de los que se compone nuestro entorno socioeconómico. Esto es, la innovación conlleva per se, en el marco actual de nuestra sociedades, una adscripción social positiva, un valor intangible, un halago del que, lógicamente, nadie está dispuesto a desprenderse.
Desde el análisis de los discursos producidos en las entrevistas cualitativas, se percibe que frente a los discursos y las etiquetas sobre la innovación que pueden rondar sobre las diferentes entidades, a la persona informante le resulta ciertamente arduo expresar con claridad qué significa, en qué consiste o cómo se materializa la actividad innovadora en el quehacer ordinario de la organización a la que representa. $Y$ a este inconveniente se le uniria, además, otro: las dificultades al delimitar qué es innovación, esto es, la ausencia de una definición clara y precisa del propio concepto de innovación a lo largo del conjunto de las entrevistas en profundidad realizadas a personas ocupadas, paradójicamente, en algunas de las organizaciones que son categorizadas como innovadoras 6 .

Tales observaciones nos conducen a considerar que las llamadas a la innovación por parte de las entidades de la C.A. del País Vasco, guardan mayor afinidad con la estabilidad y el mantenimiento de la posición que con la ruptura y la destrucción -aunque sea creativa-. Por todo ello, este trabajo de campo ha permitido corroborar que la innovación también permite a las entidades hallar un recurso de ligazón intraorganizacional, cohesionándose en torno a una actitud grupal que interioriza como parte de su cultura la necesidad estratégica de innovar, de anticiparse a las demandas no satisfechas en el entorno en el que se desarrollan su actividad. La innovación parece estar siendo válida a estas organizaciones como un discurso o paradigma para mantener la tensión interna, para no dormirse en los laureles, para estar al tanto de las dinámicas y transformaciones del entorno o contexto socio-estructural en el que se desenvuelven?.

\subsection{Los contextos socio-estructurales de la innovación}

Tal y como ya hemos destacado, las actividades de las entidades analizadas en este estudio no pueden entenderse como praxis aisladas, plegadas sobre sí mismas, sino necesariamente enmarcadas en un entorno e imbricadas en un entramado socio-estructural en el que se gestan, con el que dialogan y sobre el que actúan. De esta manera, la praxis de la innovación no puede sino entenderse desde una estrecha interdependencia entre las propias entidades y los recursos, posibilidades y limitaciones que encuentran en su entorno más cercano, proporcionadas 
por organismos públicos, otras entidades, agentes sociales y personas.

De esta manera, las posibilidades que las diversas entidades han encontrado para afianzar la praxis innovadora surgen de la interacción con el entorno más cercano, donde se adquieren los nutrientes principales que permiten descubrir nuevos terrenos y oportunidades ${ }^{8}$. Estos terrenos surgen de la interacción y de los ámbitos de relación con otras entidades y sectores. Los mecanismos de aprendizaje utilizados en estos procesos implican, no solamente la adaptación y asunción de nuevas prácticas y formas de funcionamiento internas dentro de cada entidad, sino la exteriorización de las mismas a la hora de cuidar de los clientes o de poner en funcionamiento nuevas medidas. Los condicionamientos socio-estructurales que impone cada entorno relacional obligan a cada entidad a adaptarse a sus nuevos requerimientos; así, como bien afirman muchas de las entidades, la innovación se convierte en un recurso y en una cuestión de supervencia ${ }^{9}$. Un recurso necesario para poder avanzar y poder reinventarse con los años.

En este sentido, la innovación acaba por convertirse en un aliado necesario. Si bien muchas de las entidades asumen de forma acrítica su condición de innovadoras y asocian dicha etiqueta a su capacidad para ser pioneras o inventoras de nuevas prácticas, también identifican de manera más subjetiva, que la innovación y la improvisación surgen del trabajo diario y del esfuerzo por enfrentarse a problemáticas que han de ser resueltas si la entidad pretende avanzar.

Dentro de esta dinámica de funcionamiento interno y externo también es preciso mencionar la importancia que los ámbitos públicos e intitucionales han tenido sobre la actividad de muchas de las entidades entrevistadas. Hemos encontrado ciertas reticencias y críticas al papel que desempeñan las instituciones públicas en la difusión y apoyo a la innovación en las distintas entidades. Sin embargo, a la hora de analizar los discursos de unas y otras encontramos que el apoyo institucional, fundamentalmente basado en ayudas económicas, ha sido utilizado por casi todas ellas ${ }^{10}$.

Esto plantea varios dilemas; en primer lugar, nos lleva a cuestionar hasta qué punto cada entidad depende única y exclusivamente de sí misma para progresar; en segundo lugar, plantea una reflexión sobre cuál es el espacio que se deja para la generación de emprendedores desligados de lo público, es decir, aquéllos que se valen por sí mismos en el enfrentamiento cotidiano a sus dinámicas organizacionales; en tercer y último lugar, nos preguntamos si las instituciones públicas son componentes estructurales básicos en nuestras dinámicas de innovación y desarrollo socio-económico.

De esta manera, los contextos socio-estructurales consiguen penetrar en las diversas entidades a la hora de condicionar e influenciar las formas de comprender y organizar las actividades internas y encarar los procesos innovadores. La puesta en práctica de la innovación pasa por la implantación de renovados modelos de trabajo, la gestión de las personas y de los recursos disponibles, siempre envueltos por factores intangibles como la confianza, la responsabilidad, la creatividad, el conocimiento, el respeto y la dedicación. De esta manera, la puesta en marcha de estrategias para fomentar la innovación, encuentran en los contextos socio-culturales internos de las empresas los propios condicionantes que delimitan su margen de acción.

En este contexto, la práctica de la innovación se asocia a la gestión de la complejidad interna de la entidad, a la improvisación por equilibrar los cambios en el modelo organizativo y a la adaptación de los diferentes agentes a un entorno de trabajo en el que emergen nuevas exigencias y potestades ${ }^{11}$. A este respecto, el día a día de la entidad que trata de fomentar dinámicas innovadoras tiene que convivir con diversas dificultades internas en la relación al desgaste que supone la improvisación, el dinamismo marcado por el ensayo y el error, la coordinación, el aprendizaje permanente, la búsqueda de acuerdos y consensos, la incertidumbre, las limitaciones de tiempo y presupuesto, así como la constante renovación ${ }^{12}$. Este desgaste puede interpretarse como un límite a la innovación que lejos de venir de dinámicas externas surge de la propia práctica continua de la misma, esto es, un agotamiento del éxito de la innovación.

\subsection{La red y la innovación}

La red ha sido reconocida por todos nuestros informantes como factor incisivo y fundamental a la hora de poner en práctica la innovación. Participar, difundir y cosechar de las diversas redes en funcionamiento se ha convertido en un elemento de primera necesidad para poder apren- 
der y adquirir nuevos conocimientos. Todas las entidades analizadas se apoyan en la red para la puesta en marcha de sus proyectos, en ella buscan otras experiencias, nuevos clientes, nuevos proyectos, la adaptación a las nuevas necesidades y elementos de innovación que pueden incorporar a sus propias experiencias. De este modo, la red se convierte en la herramienta principal que permite la visualización de la entidad y el espacio social que posibilita la apertura de nuevas puertas, tanto en el establecimiento de nuevas relaciones, como en los procesos de aprendizaje y transferencia de conocimiento.

Entre las nuevas oportunidades que posibilita el establecimiento de conexiones y relaciones, cabe destacar en primer lugar, que la red permite construir un contexto de colaboración al que se puede acudir en busca de nuevos clientes, en busca de información y de nuevas oportunidades para desarrollar nuevas actividades y evolucionar en las tareas propias ${ }^{13}$. En segundo lugar, la red se convierte en un ámbito de relación y de intercambio de información constante dentro del cual se difunde información, se imitan procesos, se aplica información, y se conocen a nuevos agentes y actores ${ }^{14}$.

En tercer lugar, la red es también un elemento de motivación que ayuda a resolver problemáticas y a buscar referentes en actividades y procesos paralelos que la entidad trata de encarar. Si una entidad tiene un problema, acude en busca de ejemplos de otras entidades o experiencias que hayan pasado por el mismo proceso, pregunta y comparte dichas experiencias haciendo de ese intercambio una forma de aprendizaje ${ }^{15}$. En cuarto lugar, la red es un factor que otorga cierto prestigio: si formas parte de una red de alta reputación, eso determina la posición en la que se enmarca la entidad, los clientes que posee, las vinculaciones profesionales a las que está expuesta, los foros a los que asiste, la calidad de sus actividades, etc. ${ }^{16}$. $Y$ en quinto y último lugar, la red es la herramienta de difusión por excelencia. Toda entidad se organiza y se da a conocer a través de ella. Por ello, la red es un componente más del entorno donde cada entidad realiza su trabajo y es a través de ella como se difunden y se conocen en otros lugares y contextos las actividades realizadas ${ }^{17}$.

De esta manera, la red se ha convertido en el principal mecanismo de aprendizaje al que recurren las diferentes entidades, para afrontar los dilemas de su propia actividad cotidiana. En ellas, se aprecia una aceleración de los procesos de imitación, renovación y adaptación creativa. En la medida en que una organización participa en el intercambio de experiencias, conocimientos e información con otros actores, está adquiriendo para sí nuevos conocimientos, está aprendiendo y está aplicando lo aprendido. La aplicación de otras innovaciones a procesos de mejora autónomos para producir más innovación es una práctica común entre las entidades entrevistadas en esta investigación. La innovación bebe del aprendizaje y la transferencia de conocimiento que aportan la imitación, la adaptación y la difusión. Sin la participación de estas dinámicas, los procesos de innovación disminuyen de forma notable sus posibilidades de reconocimiento y valorización.

\subsection{Las paradojas de la innovación}

Las entidades y organizaciones analizadas en este estudio demuestran que una cosa son los discursos sobre la innovación, que en fechas recientes han adquirido una presencia y protagonismo insólito en los ámbitos empresarial e institucional, y que otra muy distinta son las prácticas innovadoras concretas que se llevan a cabo. Mientras el discurso viene envuelto en bondades y asociado a la necesidad, las entidades son conscientes de que la puesta en marcha de iniciativas, proyectos y prácticas innovadoras está plagada de dificultades y problemáticas concretas ${ }^{18}$. Asimismo, consideran que la utilización del concepto no siempre es el reflejo de lo que ocurre en realidad y que utilizar el lenguaje de la innovación no convierte automáticamente a las diversas entidades en innovadoras.

Desde el momento en el que tratamos de establecer el nivel de correspondencia entre los discursos sobre la innovación y las prácticas concretas de las entidades, resulta revelador que nuestros informantes relacionen constantemente la innovación con un proceso alargado en el tiempo en el que han tenido que ir superando diferentes barreras y dificultades. Por ello, la innovación es relacionada con los procesos de adaptación y reinterpretación que se han sucedido en el seno de la entidad, en un recorrido en el que han tenido que "buscarse la vida".

En un momento en el que el discurso sobre la innovación es asociado a conceptos tales como ruptura, novedad o 
invención, el análisis de las entrevistas en profundidad permite visualizar que la innovación es un recorrido donde prevalece el trabajo persistente, sobre todo en el tratamiento de la información, la evaluación, la inquietud y el aprendizaje. En este contexto, la práctica innovadora no es solamente un resultado puntual y concreto fruto de mentes privilegiadas que operan en un contexto de neutralidad, sino fruto del trabajo constante y los procesos de transferencia de conocimiento. Según se desprende de las reflexiones realizadas por nuestros informantes, la práctica innovadora es un recorrido plagado de constancias y de rutinas, en el que lo importante es el día a dia ${ }^{19}$.

Las organizaciones participantes en esta investigación son, por lo tanto, conscientes de que la innovación se debe traducir en prácticas cotidianas y constantes en el seno de la organización, sin las que conseguir resultados resultaría una auténtica quimera. Así, para que emerjan proyectos e iniciativas innovadoras es necesaria una reorientación de las formas de trabajo y de colaboración entre los diferentes agentes involucrados en las diferentes tareas. Por lo tanto, la práctica innovadora es también el resultado de la expansión y de la socialización de una nueva cultura de trabajo y de la dedicación a las tareas, un proceso con múltiples derivas y plagado de contingencias. La innovación también nos habla de un cambio de valores, que sobre todo en el ámbito empresarial, pero también en los ámbitos social y cultural trata de aglutinar un nuevo espiritu de trabajo y de mentalidad en relación a la confianza, la dedicación, el esfuerzo, la entrega, la responsabilidad, la flexibilidad, la adaptación, el aprendizaje y la cooperación ${ }^{20}$.

Sin embargo, y a pesar de los esfuerzos realizados desde las entidades en la elaboración de estrategias para fomentar dinámicas innovadoras, el éxito inmediato no está asegurado, sino que es descrito como un proceso largo y costoso en el que constantemente se generan fricciones, desconfianzas y malentendidos ${ }^{21}$. En definitiva, la extensión de la práctica innovadora en el seno de las entidades y los procesos afines a la misma, como la participación o la colaboración exigen tiempo y esfuerzos por parte de todos los agentes involucrados. El conjunto de las organizaciones consultadas asume que la innovación sólo es posible con una adaptación constante de los hábitos y de las fórmulas de trabajo, aunque también destacan que en numerosas ocasiones se encuentran involucrados en un contexto cotidiano que les dificulta dedicar el tiempo y el esfuerzo necesario a conseguir este objetivo.

\section{Conclusiones}

El análisis de los discursos de las personas que han participado en las veintiséis entrevistas en profundidad realizadas en esta investigación sobre el modo en el que integran la praxis innovadora a la actividad cotidiana de sus organizaciones supone, sin duda, un notable contrapeso al discurso afirmativo y positivo con el que viene siendo difundida, prácticamente en exclusiva, la innovación. En el tránsito del discurso a la acción concreta, las personas informantes revelan las múltiples paradojas, dificultades, imprevistos y fluctuaciones que existen en los intentos de institucionalización de la innovación. Teniendo en cuenta este planteamiento central, a continuación presentamos las ideas principales extraídas del análisis de estas entrevistas.

a) Innovación: asunción acrítica, etiqueta no problematizada. Se constata una asunción acrítica o no problematizada por parte de nuestros informantes de la innovación, sobre todo si el término es asociado a la actividad de la entidad a la que se representa. Sin embargo, los entrevistados muestran mayores dificultades a la hora de explicar con claridad qué es lo que convierte a las entidades en innovadoras y cuáles son las medidas concretas que permiten institucionalizar la innovación. De esta manera, obtener la etiqueta de entidad innovadora resulta tan estimulante y gratificante como poner en práctica la innovación.

b) Afinidad entre innovación y supervivencia. Entre las entidades entrevistadas, la innovación es identificada con prácticas que son habituales en el desarrollo diario de cada entidad y que, por tanto, llevan efectuándose durante años, quizá antes incluso de que el término innovación tuviese connotaciones tan positivas y estuviese tan generalizado. En esta lógica, las personas informantes indican que el propio hecho de la existencia de la organización es, en sí mismo, indicador suficiente para demostrar su capacidad de adaptación a un medio o entorno hostil. La innovación es, de este modo, concebida como estrategia de supervivencia, de adaptación permanente. 
c) Innovaciones situadas o contextualizadas. Como hemos venido argumentando, los procesos de las organizaciones innovadoras se encuentran enmarcados en contextos socio-estructurales específicos. Ello implica que hemos de aprehender la práctica de la innovación en relación y diálogo con el entorno desde el que se gestan, en el que evolucionan y en el que, en ocasiones, con grado diverso transforman o buscan transformar en diferentes intensidades. Las entidades no son islas, ni realidades aisladas, sino agentes que emergen desde un contexto específico con el que interactúan de forma constante.

d) Afinidad entre innovación y estabilidad. El trabajo empírico cualitativo efectuado en esta investigación ha hallado que la innovación lejos de identificarse únicamente con la ruptura, aparece en múltiples ocasiones asociada a la búsqueda de estabilidad o pervivencia. De modo que estando presentes ciertas rupturas ante modos de hacer o modos de organización, desde un plano interno o intraorganizacional la innovación es concebida y desarrollada principalmente por parte de las organizaciones como un recurso para la estabilidad, como un mecanismo de supervivencia, como una estrategia de renovación permanente. La innovación parece estar siendo válida a estas organizaciones como un discurso o paradigma para mantener la tensión interna, para no dormirse en los laureles, para estar al tanto de las dinámicas y transformaciones del entorno o contexto socioestructural en el que se desenvuelven.

e) Afinidad entre innovación y financiación pública. Una de las cuestiones que se ha mostrado con absoluta claridad en el análisis de las organizaciones participantes, todas ellas enraizadas en ámbitos de actividad diversos, hace referencia a la importancia de la financiación pública. La transversalidad de este hecho no hace sino reforzar la idea de que, en líneas generales, hemos de entender la innovación de la mano de la financiación pública. $Y$ debemos hacerlo en dos sentidos, tanto como apoyo y cobertura a las iniciativas de las diversas entidades, como en su dependencia hacia lo público.

f) Afinidad entre innovación e imitación-adaptación creativa. Las entidades entrevistadas mencionan la importancia de tomar ejemplos y retroalimentarse de las actividades y procedimientos que ponen en marcha otras entidades con actividades parecidas a las suyas. Dichas estrategias no son, en términos estrictos, copiadas, sino más bien adaptadas a las propias realidades, con la finalidad de redirigir y mejorar muchas de las actividades de las organizaciones. Dicho de otra manera, la gran mayoría de los procesos de innovación acaban siendo procesos de imitación, lo que hace preguntarnos por el carácter o sentido de la propia innovación.

g) Innovación y persistencia de las paradojas. La práctica de la innovación no es el bálsamo que alivia todos los problemas, sino una praxis que también genera descontentos, desligados, malentendidos, desconfianzas y desentendimientos. Por todo ello, la mayor de las dificultades con las que se encuentra la institucionalización de la innovación en las diferentes entidades es en la extensión entre las personas y las propias organizaciones de una cultura innovadora fundamentada en la revisión constante de sus acciones, direcciones y soportes.

De esta manera, podemos apreciar que más allá de los discursos difundidos sobre la innovación, su práctica concreta está atravesada por múltiples circunstancias socio-culturales que participan de forma activa en su representación y devenir. Por ello, los resultados genéricos aquí resumidos se complementan con el análisis de la percepción social de la innovación entre la ciudadanía de la C.A. del País Vasco, desde el que indagar en la representación y significación que entre los ciudadanos adquieren los discursos y praxis sobre la innovación que observan y perciben a su alrededor.

\section{NOTAS}

Recibido: 24 de junio de 2010

Aceptado: 25 de octubre de 2010
1 Dicha investigación ha sido financiada por el Departamento de Industria, Innovación Comercio y Turismo del
Gobierno Vasco en el marco del programa SAIOTEK, en las convocatorias del año 2008 y 2009.

2 Los recientes trabajos de Javier Echeverría $(2008 ; 2010)$, nos permiten 
visualizar el recorrido reciente realizado por el concepto de innovación comenzando por las primeras concepciones plasmadas en el Manual de Oslo, las revisiones que sobre dicha conceptualización se han realizado y finalizando con las nuevas perspectivas sobre la innovación social y cultural defendidas por importantes agencias como las británicas National Endowment for Science, Technology and the Arts (NESTA), o Young Foundation, amén de diversos grupos de investigación y autores dedicados al análisis de la innovación desde perspectivas sociológicas, históricas o filosóficas.

3 Para Edward Soja (2008: 32), uno de los geógrafos más destacados en el análisis de nuestro tiempo histórico global, las ciudades y regiones son los espacios por antonomasia donde se observan las dinámicas innovadoras. Desde su perspectiva, nuestras acciones y pensamientos modelan los espacios que nos rodean, pero al mismo tiempo los espacios y lugares en los cuales vivimos son producidos colectiva o socialmente y conforman también nuestras acciones y pensamientos. Esta interacción entre lo construido y la capacidad humana de remodelar lo existente, que el profesor Soja denomina como "sinecismo" está especialmente extendida en las ciudades y regiones. «Tanto sinecismo como metrópolis pueden ser consideradas descripciones estáticas de la forma espacial, pero también como el contexto específicamente espacial de procesos activos y afectivos de formación, innovación, desarrollo, crecimiento y cambio sociales (...). El sinecismo, en tanto fuerza activa y motriz de la geohistoria, supone la formación de una red regional de asentamientos nucleados y anida- dos de modo jerárquico, capaces de generar innovación, crecimiento y desarrollo social (así como también individual) desde el interior de su dominio territorial definidon.

4 Tal y como relata uno de los informantes, "la innovación es una cuestión de supervivencia. Porque si seguimos haciendo lo mismo que haciamos en 1983, pues estamos muertos. ¿Queremos estar muertos? No. ¿Tenemos vocación de supervivencia? Sí ¿La gente va a seguir teniendo el problema? Sí. ¿Qué hay que hacer? Ser excelente, innovar por supervivencia (...) no innovar por innovarn (Entrevista personal 24: ámbito social).

5 Uno de nuestros informantes destaca la forma de interiorización del discurso de la innovación: "Hemos tenido una revisión de la misión, y de lo que definimos por misión en la que participó toda la gente. Hay una frase que salió y es que 'somos innovación'. Somos una empresa que estamos constantemente haciendo cosas nuevas, que nos motiva hacer cosas nuevas» (Entrevista personal 5: ámbito tecnológico).

6 El mismo informante muestra cierta incomprensión sobre la diferencia que implica la innovación con respecto a otras estrategias y conceptos aplicados con anterioridad: "Hace cuatro años estaban de modas las ISOs, se hablaba de la mejora continua. Pero en el 2004, 2005 estaban de moda las FQM, las ISOs, todas las empresas teníamos que tener ISOs de calidad porque estaba de moda en aquella época. Ahora ya nadie habla de calidad, sino de innovación. Yo muchas veces me he preguntado: ¿Qué diferencia hay entre la mejora continua de una ISO y la innovación?» (Entrevista personal 5: ámbito tecnológico).
7 Tal y como pudimos recoger en una de las entrevistas realizadas: "La idea de innovar va muy unida a la anticipación de las respuestas. Tenemos que ser capaces de leer en el colectivo al que nos dirigimos y en la sociedad en su conjunto, por donde van a ir las necesidades" (Entrevista personal 26: ámbito social).

8 Tal y como ilustra una de las entidades entrevistadas, "el tejido industrial vasco ha facilitado eso porque más que haber pocas empresas grandes, habia muchas y pequeñas y eso ha permitido que haya también a su vez unas cuantas de informática que han ido creando pues bueno, profesionales y al final hay un entramado que entendemos que es de alta calidad y eso se muestra en que fuera de Euskadi vendemos con una cierta facilidad" (Entrevista personal 9: ámbito servicios).

9 "La innovación es una cuestión de supervivencia. Porque si seguimos haciendo lo mismo que haciamos en 1983, pues estamos muertos. ¿Queremos estar muertos? No. ¿Tenemos vocación de supervivencia? Sí. ¿La gente va a seguir teniendo el problema? Sí. ¿Qué hay que hacer? Ser excelente, innovar por supervivencia (...) no innovar por innovar" (Entrevista personal 24: ámbito social).

"Creo que el concepto de innovación existe y ha existido toda la vida. Por ejemplo, el que fabricaba sobres para cartas tuvo una época en la que vendía un montón, y luego cuando llegó el e-mail, dejó de vender sobres. Pero igual se reconvirtió y se dedicó a hacer yo qué sé qué. Pero eso más que innovar es sobrevivir. Lo que pasa es que eso se ha sabido siempren (Entrevista personal 5: ámbito tecnológico). 
10 Las afirmaciones aportadas por nuestros informantes son reveladoras a este respecto: "Me gustaría añadir que la innovación, incluso para empresas como nosotros, hoy en día en España pasa por el soporte y la ayuda pública. Si es importante en nuestro caso, lo es mucho más en el caso de cualquier otra área tecnológica» (Entrevista personal 4: ámbito tecnológico).

El soporte de las instituciones públicas, se hace notar además en los diversos ámbitos en los que está presente la innovación: "Los proyectos de investigación en general son apoyados por entidades públicas, en ese sentido siempre hemos pensado y hemos mirado de una forma natural que las instituciones públicas apoyaran» (Entrevista personal 15: ámbito cultural).

11 Tal y como destaca uno de nuestros informantes: "Antes el 100\% de la prima era por productividad, y luego se pasó a pagar el 60\% de la prima por productividad y el $40 \%$ por calidad. Se trató de buscar el equilibrio entre ambas. Se trató de cambiar la mentalidad de las personas que estaba orientada a producir y producir y producir, y que con el apoyo de la prima y la formación intensa que se dio en aquellos momentos, se diesen cuenta de que no solamente había que hacer las piezas sino que había que hacerlas bien» (Entrevista personal 1: ámbito industrial).

12 Uno de nuestros informantes asume con normalidad este hecho cuando apunta que: "Hay un componente de incertidumbre bastante alto. Eso nos produce una problemática a la hora de planificar producción. Es muy difícil que yo tenga a toda la gente al 100\% trabajando todo el año, eso es imposible. Hay veces que estamos al $100 \%$, incluso haciendo horas extras, pero luego hay épocas en las que están al 60\%" (Entrevista personal 5: ámbito tecnológico).

13 Las afirmaciones de uno de nuestros informantes son reveladoras en este punto: "En el equipo que formamos vemos oportunidades de colaboración, de desarrollar ciertas cosas. Pertenecemos a las empresas llamadas TICs, a ese cluster, somos empresas que desarrollamos soluciones para los demás, benefician a los demás que están en sectores muy diferentes a los nuestros: automoción, energía, modificación y construcción. Tratamos de estar en todos los foros. Existen reuniones trasversales en el sector intra sector donde solemos estar nosotros. Tratamos de ver que demandan..." (Entrevista personal 5: ámbito tecnológico).

14 Tal y como destaca uno de nuestros informantes al respecto: "Nosotros pertenecemos a redes, esa pertenencia supone el $60 \%$ de los procesos de vigilancia tecnológica, es un beneficio de cara a la investigación» (Entrevista personal 24: ámbito social).

15 El recurso de aprendizaje que aportan las experiencias paralelas es práctica común entre las entidades entrevistadas: "Alguien que hace los mismo que tú, que tiene los mismos problemas que tú, las mismas dificultades, los mismos logros, es importante compartirn (Entrevista personal 15: ámbito cultural).

16 Uno de nuestros informantes revela que además de la visualización, la red ofrece una distinción que puede beneficiar a las actividades de la entidad: "Al conocerte porque formas parte de algo, de una red, estamos teniendo y obteniendo demanda de los diferentes colectivos sociales" (Entrevista personal 24: ámbito social).
17 Uno de nuestros informantes destaca que el potencial de difusión de la actividad de la entidad se encuentra en la creación de redes y en el aporte en los mismos, cuando afirma que "uno sólo no puede cambiar la sociedad, es preciso hacerlo acompañado de otros muchos" (Entrevista personal 22: ámbito social).

18 Tal y como ponía de manifiesto uno de nuestros informantes: "Innovación. Vale. ¿Y ahora qué? Seguimos de la misma manera, con las mismas cosas y no nos movemos ni un ápicen (Entrevista personal 24: ámbito social).

19 «(...) pero lo importante aquí es el día a día, el día a día y la idea puede surgir hoy, uno dice "uy, y por qué no hacemos eston y eso que puede ser una conversación en un café pues termina en una gran idea, y en un gran producto. Entonces eso, eso es continuo, y sale de cada una de las personas que hay aquín (Entrevista personal 9: ámbito servicios).

20 "Confianza en los trabajadores, formación total, que entiendan cual es el contexto del trabajo que están haciendo y asumir que muchas veces, detrás del trabajo que hago yo, no hay nadie vigilando. Aunque sea el almacenero. Si no hace una entrega correcta, tiene que saber el impacto que tiene. Insisto. No es una estrategia para ahorrar costes. De la otra forma estarias en un mercado en el que no serias competitivo" (Entrevista personal 3: ámbito tecnológico).

21 «Para algunos es demasiado vivo porque esa sensación de estar en un cambio continúo, si no entiendes la naturaleza y el porqué, puedes llegar a percibir que no se sabe muy bien lo que se hacen (Entrevista personal 3: ámbito tecnológico). 


\section{BIBLIOGRAFÍA}

Chesbrough, H. W. (2009): Innovación Abierta, Barcelona: Plataforma.

Echeverría, Javier (2008): "El manual de Oslo y la innovación social", Arbor: Revista de Ciencia, Pensamiento y Cultura, n. 732, pp. 609-618.

- (2011): "Ocho Propuestas sobre la Innovación Social", en A. Gurrutxaga y J. Echeverría (eds.), La Luz de la Luciérnaga: Diálogos de Innovación Social, Madrid, Plaza y Valdés, pp. 67-109.

Gurrutxaga, Ander (2007): "Cambio, Innovación, Complejidad y Orden Global", en A. Gurrutxaga (ed.). Retratos del Presente: La Sociedad del Siglo XXI, Bilbao, Universidad del País Vasco, pp. 53-84.

- (2010a): Recorridos por el Cambio, la Innovación y la Incertidumbre. Bilbao: Universidad del País Vasco.

- (2010b): "Diálogo con la Innovación", en La Luz de la Luciérnaga: Diálogos de Innovación Social, Bilbao, ASCIDE, pp. 13-65.
Hämäläinen, T. (2007): "Social innovation, structural adjustment and economic performance", en T. Hämäläinen y R. Heiskala (eds.), Social Innovations, Institutional Change and Economic Performance. Making Sense of Structural Adjustment Processes in Industrial Sectors, Regions and Societies, Edward Elgar Publishers, Cheltenham, pp. 11-51.

Klein, Juan-Luis y Harrison, Denis (2006): L'Innovation Sociale: Émergence et Effets sur la Transformation des Sociétés. Québec: Presses de l'Université de Québec.

Lester, R. K. y Piore, M. J. (2004): Innovation. The Missing Dimension, Oxford: Oxford University Press.

MacCallum, Diana; Moulaert, Frank y HiIlier, Jean (2009): Social Innovation and Territorial Development, London: Ashgate.

Moulaert, Frank y Nussbaumer, Jacques (2005): "La Región Social: Más Allá de la Dinámica Territorial de la Econo- mía del Aprendizaje", en Ekonomiaz: Revista Vasca de Economía, n. ${ }^{\circ}$ 58, pp. 96-127.

NESTA (2007): Hidden Innovation, London, NESTA.

Saxenian, Anna Lee (1994): Regional Advantage: Culture and Competition in Silicon Valley and Route 128, Cambridge: Harvard University Press.

Soja, Edward W. (2008): Postmetrópolis: Estudios Críticos sobre las Ciudades y las Regiones, Madrid: Fabricantes de Sueños.

Von Hippel, Eric (1988): The Sources of Innovation, New York: Oxford University Press.

Young Foundation (2007): Social Innovation: What Is It, Why It Matters, How It Can Be Accelerated, London: Basingstoke Press.

- (2008): Breakthrough Cities: How Cities Can Mobilise Creativity and Knowledge to Tackle Compelling Social Challenges, London, British Council. 